Editorial

\title{
Covid-19: Critical Lessons for the Survival of Mankind from the Present and Future Pneumonic Viral Infections
}

\author{
K. K. Sharma ${ }^{1} \quad$ J. N. Pande ${ }^{2}$ \\ ${ }^{1}$ Department of Pharmacology, University College of Medical \\ Sciences (University of Delhi) \& GTB Hospital, Delhi, India \\ ${ }^{2}$ Sitaram Bhartia Institute of Science \& Research, New Delhi, India
}

Ann Natl Acad Med Sci (India) 2020;56:1-5

\author{
One who wins without problems, it is just \\ "VICTORY," but, \\ One who wins with lot of troubles, it is "HISTORY".
}

Covid-19, the short for Coronavirus Disease (occurring in) 2019, hit the world with a bang, beginning from Wuhan, the capital of Hubei province in China. Tracing the history, the first case (P-zero) was detected on December 8, 2019 (now recognized to be a woman of 57 years, who sold shrimps in the Huanan market admitted in the Wuhan Union Hospital on December 10 and diagnosed on December 31 with Covid-19) before several workers from the Huanan seafood market started to fall sick with fever and respiratory symptoms of shortness of breath, sneezing and cough, and some with dyspnea and signs of pneumonitis., ${ }^{1,2}$ Three weeks later on December 31, Wuhan Health Authorities announced a series of 21 cases of suspected pneumonia of unknown origin, all linked to seafood market, and notified to the World Health Organization (WHO) country office in China. ${ }^{2,3}$ The disease appeared to be highly infectious, causing considerable anxiety among physicians. Within a month, 41 patients were getting treatment for this unique infection in the Wuhan Union Hospital, where one of them died in the meantime (but the P-zero is still surviving). Due to fast-spreading transmission events, Covid- 19 cases were widespread not only across mainland China but also beyond Chinese borders by January 21, 2020, as a result of symptomatic/ asymptomatic international or national travelers returning to their native countries or other states of China, respectively. On January 30, 2020, the International Health Regulations Emergency Committee (IHREC) of WHO declared the disease epidemic as Public Health Emergency of International Concern (PHEIC), because as of January 27, more than 2000 cases had been diagnosed in several other countries, besides China and Hongkong: Thailand, Japan, South Korea, Singapore, Vietnam, Taiwan, Nepal, Macao, Australia, France, Canada, and US. Most of these patients were living in, visited Wuhan, or were their contacts. ${ }^{1-3}$ Human-to-human transmission was confirmed. ${ }^{4}$

\section{China's Response to Covid-19 Pandemic}

China's response to Covid-19 was quick; by early January, 2020, the virus was identified and genomic sequence determined. ${ }^{5}$ The virus was earlier termed 2019-nCoV (n for novel) but finally named SARS-CoV-2 (as well as the disease caused by it as Covid-19) on February 11 , because it shared $79 \%$ identity with severe acute respiratory syndrome (SARS) Coronavirus (SARS-CoV/ SARS-CoV-1) and 50\% identity with Middle East respiratory syndrome (MERS) Coronavirus (MERS-CoV). ${ }^{5,6}$ Besides, it was also revealed that it shares $88 \%$ sequence identity to two Coronaviruses found in bats, batSL-CoVZC45 and bat-SL-CoVZXC21. ${ }^{6}$ Based on the fact that RNA Coronavirus typically evolves at a rate of $10^{4}$ nucleotide substitution per year, it was suggested that this virus has emerged from a single source in early December or late November 2019. It was also confirmed that eight complete genomes of virus collected from the nasal/nasopharyngeal and broncho-alveolar lavage (BAL) fluid samples of first cohort of patients were 99\% identical. Initially, epidemiology showed that the virus spreads directly via contaminated aerosolized droplets exuded from coughing and sneezing and inhaled during inspiration by the person coming in contact of the infected patient, and indirectly via contaminated hands and surfaces through touch and contact of facial skin near nose; extensive person-to-person transmission was confirmed, with clusters of infected families and close contacts of P-zero (P0 or index case) and medical staff. ${ }^{7}$ There are reports that virus could be transmitted even before the contagion-positive person shows symptoms. ${ }^{3,4}$ Soon it became evident that the rapid rise of confirmed
Address for correspondence

K. K. Sharma, A-5, Parivahan

Apartments, Sector 5, Vasundhara,

Ghaziabad 201012, Uttar Pradesh, India

(e-mail: drkksharma2000@yahoo.com).
DOI https://doi.org/

10.1055/s-0040-1710443 ISSN 0379-038X.
C2020 National Academy of Medical License terms

Sciences (India) 
cases outstripped the rate of increase of cases of SARS in $2002 / 3$, raising serious global health concerns.

The super transmission events followed over the course of the next 3 weeks: by March 11, the number of cases of Covid-19 outside China increased by 13 -fold, that is, more than 1,18,000 and number of countries tripled, that is, 114 nations with 4291 people losing their lives to the disease, forcing the WHO to declare it as a pandemic. ${ }^{8}$ The decision was not taken to create panic but to take quick, timely, informed, and decisive measures in order to contain the disease before it threatens the total survival of humanity and overwhelms the systems which support it at risk, be it health, economy, environment, and so on. In legal terms, characterizing the Covid-19 as pandemic made the whole world implement the measures required to contain the disease effectively, as per laws of the land.

As the Covid-19 is a new disease and still evolving, its epidemiology, transmission mode and rate of progression, pathophysiology, phenotype and clinical features, major predisposing risks, and treatment options are not fully understood. Whatever little understanding we have about this disease was as a result of our experiences with similar SARS or SARS-like diseases due to SARS-CoV emerging in 2002-2003, MERS caused by MERS-CoV in 2012, and lessons learned from the outbreak of H1N1 influenza pandemic of 2009. Therefore, characterizing Covid-19 as pandemic would make all the affected countries comprehensively implement strict measures of geographical (inter- and intracountry, inter- and intraregion and inter- and intracity, etc.), community, cluster, and individual social/physical isolation by strict lockdown and sealing of areas/territories, suspend transport and travel, and quarantine the suspected asymptomatic patients/contacts of infectious patients to reduce its transmission and expansion at the local and global level.

In the initial weeks following the spread of virus, the Chinese government enacted laws and implemented draconian quarantine and social/physical isolation and clinical treatment measures, and ordered strict travel restrictions, suspending national and international travel and forcing lockdown of states and cities, which affected normal life activities and movement of hundreds of millions of citizens. In some hard-hit cities, residents were unable to leave their homes for more than a month, while transport between major population hubs has been limited or halted altogether. Although, these unprecedented measures imposed a steep toll, both on many millions of ordinary citizens who were forced to endure a life under lockdown, and country's economy witnessing a nosedive in the following weeks, it had a salutary effect in slowing and ultimately halting the progress of the Covid-19 in the country.

China's pathbreaking, proactive, and systematic risk management strategies can be defined by $4 \mathrm{Rs}$, that is, Right decisions at Right time and Right place for the Right people have proven to be effective in containing and controlling Covid- 19 . This became possible because of collaboration between the government officials and health experts, paying attention to scientific evidence, and listening to local and international health scientists. ${ }^{8}$

One can fight the virus once one knows where it is. The weaponry used in this fight consisted of 5Ts, that is, Trace (and identify people who are exposed to pathogen), Test (them for SARS-CoV-2, and if found positive but asymptomatic, isolate them and put in quarantine as they are at high risk to infect others), Treat (them if they are symptomatic as per national/global guidelines), Track (the people, patients and clusters/hotspots of disease by carrying out all-round monitoring to prevent the further spread of outbreak), and create an atmosphere of coordinated Teamwork to obtain every one's cooperation to win the situation.

As of Tuesday, the March 31, 2020, the global tally of SARS-CoV-2 cases crossed the 8,00,000-mark (which also included 1,65,659 cases who have recovered/discharged from hospitals) with 40,000 reported deaths and affecting 200 countries and territories, besides the two international conveyances: the Diamond Princess Cruise Ship (harbored in Yokohama, Japan) and America's MS Zaandam Cruise Ship (harbored in Holland). Hard-hit Italy, Spain, and Iran now account for more than half of all Covid-19 cases and deaths worldwide, and US has the most confirmed cases. The death toll of 34,000 fatalities in the US from Covid-19 has surpassed the official tally of 33,000 deaths in China, where the disease now is in the phase of recovery.

\section{Global Handling of Covid-19 vis-a-vis Other Respiratory Tract Viral Infections}

Several coronaviruses are pathogenic to humans but present with mild symptoms. However, SARS-CoV-2 is the 3rd highly pathogenic coronavirus to emerge in the last two decades. The first coronavirus outbreak was of SARS in November 2002 which originated in animal food market in Foshan, a city in south-west of Gangzhou in the Guangdong province of China. This outbreak accounted for 8,422 cases of SARS with 916 deaths across 29 countries (5,300 cases and 349 deaths in China alone), with an estimated case fatality of $11 \%{ }^{9}$ MERS was the second coronavirus disease which occurred in 2012 in Saudi Arabia and other Middle East countries. It led to 2,494 cases and 858 deaths; an additional 38 fatalities were reported later from South Korea, with a total of 27 other countries reporting the cases of MERS. ${ }^{9}$

Like SARS-CoV-2, SARS, and MERS, coronaviruses are found to originate from bats and transmitted to humans through intermediate hosts, Himalayan civet cats and dromedary camels, respectively. In case of Covid-19, the first measure taken by the local authorities in China was to alert the WHO within 4 weeks of detection of the first patient and also close the Huanan market with the aim to further prevent any zoonatic transmission. This is in complete contrast to the SARS outbreak in 2002 when it took China 5 months to report the disease to WHO and identify the civet as a reservoir for the disease and in the mean time the cat continued to be sold in food markets throughout the country.

\section{Clinical Manifestations and Fatality Rate}

The clinical data from the 55,924 laboratory confirmed SARS-CoV-2 positive patients showed the following common symptoms: fever (98\%), dry cough (76\%), myalgia and fatigue 
(44\%), breathlessness (31\%), sore throat (rarely), sneezing, stuffy nose and flu-like symptoms (5\%); the majority of them (75\%) showed bilateral pneumonic changes on CT imaging. Eighty-one percent of cases were considered mild and only 1 to $2 \%$ asymptomatic. ${ }^{8-10}$ The mortality rate was initially estimated to be $15 \%$, although on the basis of the currently available data on large number of patients from several countries, the fatality rate could be as high as 1 to $2 \%$ of all infections. Critical illness in severe cases had respiratory failure, septic shock, and multiorgan failure. The disease seems to have a predilection for the elderly male population, especially with comorbidities like diabetes mellitus, cancer, hypertension, cardiovascular and chronic respiratory diseases in whom disease severity was observed to be high. Infants and children appeared to suffer less as compared with adults. Children recorded no fatalities, but they can infect adult patients who come in their contact. Thus, all contacts of infant/ child patient need to take all precautions from catching infection from children with Covid-19 infection. The average incubation period is around 5 to 6 days but it appeared to be quite variable and may be as long as 14 days. ${ }^{11}$

\section{Patient Management}

Equipped with the previous experience, knowledge and lessons from the SARS and MERS outbreaks, it was not only easy to identify the novel pathogen using whole genome sequencing and to develop PCR-based diagnostic tests RT-PCR and its variants from the genomic data but also get much needed international cooperation once the WHO was informed in time. As the disease has a super-spreading characteristic, a WHO-guided key strategy to reduce transmission was used, which involved correctly triaging and identifying patients with severe acute respiratory infection at first point of contact to minimize exposure to others. The epidemiological and clinical criteria must be met to classify the cases. Currently, any one with severe respiratory infection, requiring hospital admission with no alternative diagnosis and a travel history to an affected country, region, or area during the 14 days before the onset of symptoms, or anyone with any acute respiratory illness and contact with a confirmed or probable case of Covid-19 (including in a healthcare facility), falls under a suspected case definition. ${ }^{9}$ These case definitions vary slightly from one country to another and may include evidence of bilateral infiltrations in chest imaging, specific symptoms of breathlessness or cough or anyone with the fever and a history of travel to the country(ies) affected by Covid-19.

With the timely release of disease-related epidemiological and clinical data in the public domain, WHO helped many countries around the world prepare better to suppress the spread and use it for the treatment of infection. For instance, analyzing the more than 40,000 cases' data in China, the world came to know that $80 \%$ of Covid-19 patients will not need medical intervention, while $20 \%$ would need medical treatment and care. This has made the health experts wise in balancing the demands of responding directly to Covid-19, while maintaining essential health service delivery undisturbed, thereby protecting healthcare workers and mitigating the risk of system collapse due to large spurt in cases suddenly. All observations in tackling the Covid-19 patients in almost all affected countries including China showed that suppression of virus transmission by social/physical distancing among the general population and social isolation for those who have, or live with someone having the disease, is a better strategy. Besides, the Covid-19 patients need to be quarantined in isolation with equal protection of all contacts, including the healthcare staff by proper use of personal protective equipments (PPEs) and observing all hand/body hygiene practices. A recent study from the Imperial College, London, using the data of population dynamics and healthcare facilities in 202 countries not only reiterated these observations but clearly showed that in the absence of suppressing interventions, Covid-19 would result in 70 billion infections and 40 million deaths globally in 2020. Mitigation strategies focusing on shielding the elderly (60\% reduction in social contact) and slowing but interrupting transmission $(40 \%$ reduction in social contacts for wider population) could reduce the burden by half, saving 20 million lives; besides, the study predicted that even in this scenario, health system in all countries will be quickly overwhelmed. ${ }^{13}$ This effect is likely to be most severe in lower-income settings where capacity is lowest. The study further projected that the mitigating scenarios will lead to a peak demand for critical care beds (as most of these patients are having acute severe respiratory syndrome due to pneumonia and needed ventilatory and allied support) in a typical low-income setting, outstripping supply by a factor of 25 , in contrast to a typical high-income setting, where this factor is 7 . As a result, it can be anticipated that the true burden in low-income setting following mitigation strategies could be substantially high than reflected by the above estimates.

\section{Potential Treatment Strategies}

Presently, there is no specific antiviral treatment recommended for Covid-19, and also no vaccine is available. The treatment is symptomatic. ${ }^{14}$ For patients with severe infection, oxygen therapy is the major treatment intervention to control hypoxia and respiratory distress. Mechanical ventilation may be required in cases of respiratory failure refractory to oxygen therapy, whereas hemodynamic support is necessary for managing septic shock. However, systemic corticosteroids therapy for viral pneumonia or acute respiratory distress syndrome (ARDS) are not recommended. It is suggested that nonselective or inappropriate administration of antibiotics should be avoided; however, some scientists recommend it.

Although, no antiviral treatments have been approved, taking lead from the hindsight and experience of drugs found effective in controlling SARS, MERS, Swine flu, Ebola, Zika infections, several approaches have been proposed, tried, some scientifically evaluated and informed to the medical community for the large good of the humanity. Some of these drugs which showed promising results include the use of two anti-HIV drug combination of protease inhibitors: lopinavir and ritonavir (that have been reported earlier to reduce SARS and MERS replication), 
remdesivir (an approved reverse transcriptase inhibitor for the treatment of Ebola infection), chloroquine, and hydroxychloroquine (anti-malarial and immunomodulating agents), all have been demonstrated in vitro anti-SARS-CoV-2 activity. ${ }^{15}$ Tocilizumab, a humanized IgG1 monoclonal antibody against IL-6 receptors and Interferon- $\alpha$, has also been shown to suppress the Covid-19 progression.

The use of hydroxychloroquine has recently been recommended by the ICMR-mandated National Taskforce for Covid-19 for use as prophylaxis of SARS-CoV-2 infection in selected individuals who are asymptomatic healthcare workers involved in care of suspected or confirmed cases or asymptomatic household contacts of laboratory-confirmed cases with a rider that the drug should not be given to known cases of retinopathy, cardiac rhythm disorder or hypersensitivity to hydroxychloroquine and those children who are less than 15 years of age. ${ }^{16}$

In addition, various vaccine strategies such as using inactivated SARS viruses, live attenuated viruses using latest codon deoptimization technology, viral vector-based vaccines, subunit vaccines, recombinant proteins, and DNA vaccine, have been developed and are being evaluated in animals. For some which have shown promising preclinical results, clinical trials have just begun, but will take months to year to become a therapeutic reality.

\section{Covid-19 Scenario in India}

In a short span of little over three months since December 2019, the Covid-19 has ravaged one nation after another and spread to more than 195 countries/territories affecting $7,85,807$ people as confirmed cases with 37,820 deaths. The worst affected are European countries like Italy, Spain, France, Germany, UK, and others like Iran and US. However, India taking cue from the experience of other countries, where the Covid-19 cases are very high, had put in place the controlling measures to prevent the spread of the disease much earlier, i.e., from March 22, 2020 starting with a 'Janta Curfew' announced by the GoI, when the number of total cases in the country were around 500 only. This was accomplished by adopting the balanced approach of more suppressive than mitigating strategies through strict lockdown; sealing of hotspot areas; and suspending the transport and travel facilities in regions, states, cities, and community areas all over the country. The people were motivated to avoid unnecessary movement, physical contact, overcrowding, and observe social distancing norms and wear face mask in public places. Additional measures were taken to obtain cooperation from general public and central and State governments' staff members irrespective of their belonging to any category of service to work hand-in-hand to defeat the Covid-19 outbreak. By March 31, India reported only around 5000 total Covid-19 cases with 150 deaths. Although the comparisons are odious, it is important to put the global data of rise of Covid-19 cases into perspective. When the data of rise in cases in India are compared with the increase in cases in United States and European countries in the same time, it would be observed that this rise in cases is far more lower than many advanced countries of the world. This is no small feat for a country with a population of more than 130 billion people, having limited economic resources but highly dedicated health professionals/workers and members of the administrative, academic, and scientific institutions. The role of such people working from various central and state government ministries' task forces in the ICMR, Niti Aayog, AIIMS, medical colleges and research institutes all over the country and large and small hospitals located both in urban/rural areas and belonging to both public/private sectors is no small achievement. These people also included the officers and staff of police and para-military forces, all working as partners in preventing the spread of SARS-CoV-2 infection. A unique situation which has been noted in the Covid-19 pandemic all over the world including India is that the police and allied forces' personnel are equally participating along with the health professionals in preventing the Covid-19 outbreak by not only enforcing the strict lockdown and sealing of local areas and hotspots of the disease but also checking the unnecessary movement of people and ensuring restrictions on transport and travel activities. Without such a participation by police and law enforcing personnel, the success of the preventive activities could not have been guaranteed. All these people worked as a cohesive team with a scientific fervor, and are the proud partners in combating the Covid-19 outbreak. Nonetheless, the story is not over yet, as we have miles to go; however, we will certainly win if dedicated work is continued on a sustained basis with the integrated and holistic approach.

In view of the size of the country and meager resources, some people are attributing the India's achievement of less cases and mortality to the genetic profile of population, universal BCG vaccination program, and an infection produced by a genomically mutated SARS-CoV- 2 with attenuated virulence. These attributes may be coincidental, but, in any case, it cannot minimize the efforts of citizens of India, irrespective of their belonging to any vocation and discipline.

No doubt, we are amid the hard times. It is time to reassure us but with a resolve of social distancing. The history of human civilization is testament to man's triumph on numerous such catastrophes. Today the virus is a formidable adversary, but soon enough, it will be tamed. We must give it time and bear the pain and loss incurred on the way with equanimity. There is no other better way. In the grand scheme of things, such calamities are bound to come and go, and the struggle to win them over will always entail a sacrifice. The same courage, scientific wisdom and medical ingenuity that enabled us control once dreaded small pox, polio, and HIV will once again deliver us to safety and make us come out as winner in our battle with SARS-CoV-2 too. This will happen slowly but definitely. However, let us not leave even an iota of doubt in our efforts and resolve.

\section{Conflict of Interest}

None declared. 


\section{References}

1 Zhou P, Yang XL, Wang XG, et al. A pneumonia outbreak associated with a new coronavirus of probable bat origin. Nature 2020. Doi: 01038/541586-020-2012-7

2 Zhu N, Zhang D, Wang W, et al; China Novel Coronavirus Investigating and Research Team. A novel coronavirus from patients with pneumonia in China, 2019. N Engl J Med 2020;382(8):727-733

3 World Health Organization. Novel coronavirus - China Disease Outbreak news: update 12 January (cited 2020 Feb 12). Available at: https://www.who.int/CSR/don/12-Jan-2020-novelcoronavirus-China/en. Accessed March 16, 2020

4 Li Q Guan X, Wu P, et al. Early transmission dynamics in Wuhan, China of novel coronavirus-infected pneumonia. N Engl J Med 2020;382(13):1199-1207

5 Corman VM, Landt O, Kaiser M, et al. Detection of 2019 novel coronavirus (2019-nCoV) by real-time RT-PCR. Euro Surveill 2020;25(3):2000045

6 Lu R, Zhao X, Li J, et al. Genomic characterisation and epidemiology of 2019 novel coronavirus: implications for virus origins and receptor binding. Lancet 2020;395(10224):565-574

7 Chan JFW, Yuan S, Kok KH. A familial cluster of pneumonia associated with 2019 novel coronavirus indicating person-to-person transmission. Lancet 2020;395(10223):514-523

8 Huang C, Wang Y, Li X, et al. Clinical features of patients infected with 2019 novel coronavirus in Wuhan, China. Lancet 2020;395(10223):497-506

9 World Health Organization. Global Surveillance for Human Infection with Coronavirus Disease (COVID-19). Available at: https://www.who.int/publications-detail/global-surveillance-for-human-infection-with-novel-coronavirus-(2019-ncov). Accessed April 18, 2020

10 World Health Organization. Middle East Respiratory Syndrome Coronavirus (MERS-CoV). Available at: https://www.who.int/ emergencies/mers-cov/en/. Accessed March 16, 2020

11 Xu Z, Shi L, Wang Y, et al. Pathological findings of COVID-19 associated with acute respiratory distress syndrome. Lancet Respir Med 2020;8(4):420-422

12 Chinese Centre for Disease Control and Prevention. The epidemiological characteristics of an outbreak of 2019 novel coronavirus disease (COVID-19)-China. Available at: https:// weeklychmacdc.cn/en/article/id/e53946e2c6e4-4-e9-9a9bfea8db1a8f51. Accessed March 16, 2020

13 Walker PGT, Whittaker C, Watson O, et al. The Global Impact of COVID-19 and Strategies for Mitigation and Suppression. London: Imperial College; 2020 1-19

14 Cascella M, Rajnik M, Cuomo A, et al. Features evaluation and treatment of coronavirus (COVID-19). In: SatPearls [Internet] Treasure Island (FL): SatPearls Publishing; 2020

15 Wang M, Cao R, Zhang L, et al. Remdesivir and chloroquine effectively inhibit the recently emerged novel coronavirus (2019-nCoV) in vitro. Cell Res 2020;30(3):269-271

16 Anonymous. Revised Guideline on Clinical Management of COVID-19. New Delhi: Directorate General of Health Services (EMR Division), Ministry of Health \& Family Welfare, Govt. of India, p. 1-18. Available at: https://www.mohfw. gov.in/pdf/RevisedNationalClinicalManagementGuidelineforCOVID1931032020.pdf. Accessed April 18, 2020 